\title{
Optimized universal color palette design for error diffusion
}

\author{
Bernd W. Kolpatzik* \\ Charles A. Bouman \\ Purdue University \\ School of Electrical Engineering \\ West Lafayette, Indiana 47907-1285 \\ E-mail: kolpatb@mr.polaroid.com
}

\begin{abstract}
Currently, many low-cost computers can only simultaneously display a palette of 256 colors. However, this palette is usually selectable from a very large gamut of available colors. For many applications, this limited palette size imposes a significant constraint on the achievable image quality. We propose a method for designing an optimized universal color palette for use with halftoning methods such as error diffusion. The advantage of a universal color palette is that it is fixed and therefore allows multiple images to be displayed simultaneously. To design the palette, we employ a new vector quantization method known as sequential scalar quantization (SSQ) to allocate the colors in a visually uniform color space. The SSQ method achieves near-optimal allocation, but may be efficiently implemented using a series of lookup tables. When used with error diffusion, SSQ adds little computational overhead and may be used to minimize the visual error in an opponent color coordinate system. We compare the performance of the optimized algorithm to standard error diffusion by evaluating a visually weighted mean-squared-error measure. Our metric is based on the color difference in CIE L*a* $\mathrm{b}^{*}$, but also accounts for the lowpass characteristic of human contrast sensitivity.
\end{abstract}

\section{Introduction}

In recent years, there has been a dramatic increase in the need for moderate and low-cost equipment to display digital color images. While many of the applications that drive this need require the highest possible quality, cost considerations often restrict color displays to 8 -bit video memory. This 8-bit re-

*Current affiliation: Polaroid Corporation, Image Science Laboratory, Cambridge, MA 02139.

Paper 94-018 received May 18, 1994; revised manuscript received Feb. 20, 1995: accepted for publication Feb. 28, 1995.

$1017-9909 / 95 / \$ 6.00$. (c) 1995 SPIE and IS\&T striction only allows the simultaneous display of 256 colors from the full gamut of $2^{24}$ possible colors. The selection of this palette of colors is then of critical importance.

There are a number of approaches for ameliorating the effects of a restricted color palette. "Palettization' techniques work by choosing a palette that best represents a particular image. ${ }^{1-4}$ This method yields high-quality results, but does not allow for the simultaneous display of multiple images. This is because the palette required for each image will be different, so the combined palette for multiple images will generally be too large. Iverson and Riskin ${ }^{5}$ have proposed a method for combining image palettes, but such a process must inevitably lead to degradation of image quality.

The alternative approach, studied in this paper, is to use an optimized universal palette. The advantage of a universal color palette is that multiple images can be displayed simultaneously, since each image uses the same palette. Unfortunately, direct quantization using a universal color palette generally yields much lower image quality than an imagedependent palette. Therefore, halftoning algorithms such as multilevel dithering ${ }^{6}$ or error diffusion ${ }^{7,8}$ must be used to improve the visual quality of the displayed images. These methods exploit the lowpass nature of the human visual system to hide color quantization artifacts.

A variety of approaches to universal color palette design have been previously studied. Goertzel and Thompson ${ }^{9}$ examined separable color palettes in RGB for use with error diffusion. They found that image quality was improved by distributing quantization levels along the three primaries based on the $L^{*}$ component of the CIE $L^{*} a^{*} b^{*}$ color space. However, the separable nature of their RGB structure restricts the optimality of the resulting palette. Alternatively, Gentile, Walowit, and Allebach ${ }^{6}$ found that a nonseparable universal color palette designed in $L^{*} u^{*} v^{*}$ coordinates gave superior performance over one designed in RGB coordinates. However, because the quantizer is not separable with respect to 
$L^{*} u^{*} v^{*}$, quantization of each color requires a computationally expensive search of the entire palette. More recently, Venable, Stinehour, and Roetling ${ }^{10}$ designed an optimized universal color palette based on uniform separable quantization of a scaled CIE $L^{*} a^{*} b^{*}$ color space. However, because the display gamut is no longer a cube in the $L^{*} a^{*} b^{*}$ color space, straightforward application of this method leads to wasted colors due to gamut mismatch. Also, use of this palette in error diffusion requires a transformation to the $L^{*} a^{*} b^{*}$ color space.

In this paper, we present a method for designing a universal color palette, which minimizes visual error and allows very fast quantization. Our approach is based on vector quantization (VQ) methods in a uniform color space CIE $L^{*} a^{*} b^{*}$, but it employs a recently developed VQ technique called sequential scalar quantization (SSQ).$^{4,11,12}$ The SSQ method uses a structured codebook to uniquely combine the performance advantages of a vector quantizer with the speed of a separable scalar quantizer. In practice, the quantization of a pixel into an SSQ palette may be implemented by using a sequence of three lookup tables; thus, a complete search through the color palette can be avoided.

To improve the subjective quality of the quantized image, we apply the optimized color palette in conjunction with a visually optimized error diffusion technique described in Ref. 13. Generally, error diffusion distributes errors at higher spatial frequencies and thereby reduces human visual sensitivity to those errors. In addition, optimized error diffusion exploits the difference in modulation transfer functions for luminance and chrominance components of color, and thus further reduces the perceived error of the displayed images.

Furthermore, we show how our optimized color palette may be efficiently combined with color error diffusion. To produce accurate color matches, the error diffusion algorithm must be performed in color coordinates, which are linearly related to intensity. Therefore, the $L^{*} a^{*} b^{*}$ coordinates of the color palette may not be used in the error diffusion filter. To eliminate the need for computationally expensive transformations, we introduce a new linear color coordinate system called $Y_{y} c_{x} c_{z}{ }^{14}$ Because this new coordinate system is carefully chosen to align with the $L^{*} a^{*} b^{*}$ system, it preserves the structure of the SSQ palette. Hence, error diffusion and SSQ quantization may be performed in the same coordinate system, thereby eliminating the additional computation of a transformation to $L a b$.

Finally, we investigate a visually weighted quality metric to evaluate the performance of our halftoning methods. Commonly, the quality of a match between two color patches of sufficiently large size is assessed by computing the color difference in the CIE $L^{*} a^{*} b^{*}$ color space. However, spatial frequency response must also be incorporated to account for the reduced visual sensitivity to the high-frequency quantization noise. To account for both of these effects, our metric is calculated by passing the original and halftoned images through a spatial filter approximating the human contrast sensitivity in luminance and chrominance. These spatial filters are applied in color coordinates, which are linear in intensity. The filtered signals are then transformed to the visually uniform space $L^{*} a^{*} b^{*}$ and the error energy is computed in each component.

We apply our optimized palette and error diffusion algorithm to a variety of color test images. For comparison, we also test separable RGB palettes and conventional FloydSteinberg error diffusion. ${ }^{7}$ Both subjective evaluation and our proposed quality metric indicate that the new method produces substantial and consistent improvement in image quality.

In Sec. 2 we describe the sequential color palette design. In Sec. 3 we combine our color palette with error diffusion using the new color space $Y_{y} c_{x} c_{z}$. Section 4 explains our visually weighted error metric, and Sec. 5 contains experimental results.

\section{Optimal Color Palette Design}

The objective of this section is to employ VQ methods to design an optimized universal color palette. Conventional VQ works by selecting $N$ code words (in our case, colors), which minimize some distance to the expected input values. We assume that the input color is specified in terms of the visually uniform color space $(L, a, b)$ (we will suppress the asterisk superscripts for notational simplicity). This coordinate system was specified by CIE as

$$
\begin{aligned}
& L=116 f\left(Y / Y_{n}\right)-16 \\
& a=500\left[f\left(X / X_{n}\right)-f\left(Y / Y_{n}\right)\right] \\
& b=200\left[f\left(Y / Y_{n}\right)-f\left(Z / Z_{n}\right)\right],
\end{aligned}
$$

where

$$
f(x)= \begin{cases}x^{1 / 3} & \text { if } 0.008856<x \leqslant 1 \\ 7.787 x+(16 / 116) & \text { if } 0 \leqslant x \leqslant 0.008856\end{cases}
$$

$(X, Y, Z$ are the standard color coordinates for a 2-deg observer, and $X_{n}, Y_{n}, Z_{n}$ specify the white point.

The Lab coordinate system was designed so that a justnoticeable difference in color corresponds to an approximately constant Euclidean distance. However, this approximate uniformity of $L a b$ only holds at low spatial frequencies because the design was based on large color patches. At higher spatial frequencies, the relative sensitivity to the luminance or $L$ component of color is much greater and the $L a b$ color space becomes nonuniform. This is particularly problematic when evaluating the quality of halftoning algorithms such as error diffusion, because artifacts generally occur at high spatial frequencies. To compensate for this effect, Venable, Stinehour, and Roetling applied a weighting factor $w>1$ to the luminance component of the distortion. Thus, the square of the distance between the two colors $L_{1} a_{1} b_{1}$ and $L_{2} a_{2} b_{2}$ will be

$D=w\left|L_{1}-L_{2}\right|^{2}+\left|a_{1}-a_{2}\right|^{2}+\left|b_{1}-b_{2}\right|^{2}$,

where $w$ is an experimentally determined constant.

A major disadvantage of conventional unstructured VQ is its computational complexity. Moreover, quantization using an unstructured palette requires a full search through the $N$ codebook entries. Recently, tree-structured VQ methods have been employed in color quantization applications to reduce computation. ${ }^{3,15-17}$ However, these methods still require $\log N$ operations per input color, which is excessive in many applications. 


\subsection{Overview of SSQ}

SSQ is a VQ method that imposes structure on the color palette to minimize computation., ${ }^{41,12}$ The SSQ works by performing scalar quantization successively on each component of an input vector. Figure 1 illustrates the method for the two-dimensional input vector $(L, a)$. First, the scalar component $L$ is quantized into $N_{1}=5$ regions denoted by the sets $S_{1}$ through $S_{5}$. These regions are formed by designing an optimal one-dimensional quantizer for the marginal density of $L$,

$p_{L}(L)=\int_{a \in M_{i}} \int_{b \in W_{i}} p_{L a b}(L, a, b) \mathrm{d} a \mathrm{~d} b$.

Next, for each region $L \in S_{i}$, a different one-dimensional quantizer is applied to the scalar $a$. Each of these quantizers is designed to be optimal for the conditional distribution of $a$ given that $L \in S_{i}$,

$p_{a}\left(a \mid L \in S_{i}\right)=\int_{b \in W_{i}} \int_{L \in S_{i}} p_{L a b}(L, a, b) \mathrm{d} L \mathrm{~d} b$.

Because each conditional density is likely to be different, we would expect each quantizer to be different. In addition, each quantizer will differ due to the varying number of quantization levels $n_{i}$ associated with each region $S_{i}$. For example, $n_{4}=4$ levels have been allocated to region $S_{4}$, whereas only $n_{5}=3$ levels have been allocated to region $S_{5}$. Note that the total number of levels allocated for both $L$ and $a$ is given by

$N_{2}=\sum_{i=1}^{N_{1}} n_{i}$.

The extension of SSQ to three dimensions is straightforward. Each region of $(L, a)$ formed by quantizing both components is denoted by $S_{i, j}$. For each set $S_{i, j}$, the third scalar component $b$ is quantized to $n_{i, j}$ levels and the total number of allocated colors is given by

$N_{3}=\sum_{i=1}^{N_{2}} \sum_{j=1}^{n_{i}} n_{i, j}$.

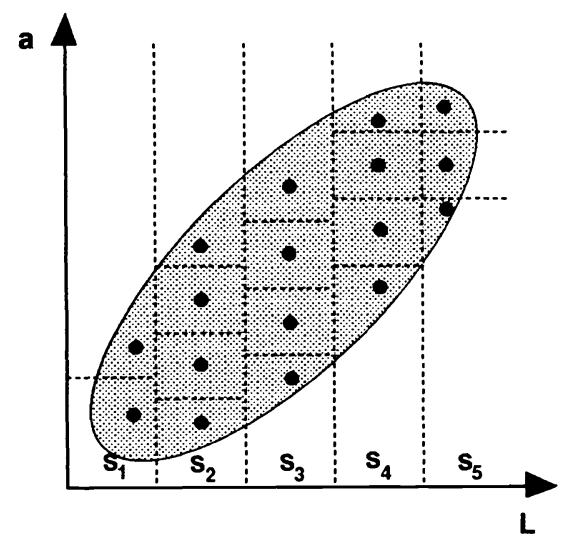

Fig. 1 Two-dimensional example for SSQ. The shaded area indicates a 2-D device gamut. Bullets denote quantization levels and dotted lines show cell boundaries. In this example, $L$ is quantized first.
Because the quantizers are different for each region $S_{i}$ or $S_{i, j}$, the SSQ method exploits the dependencies among scalar components. For example, combinations of $L$ and $a$ that fall outside the gray area of Fig. 1 are not in the device gamut and need not be quantized. We note that the order of quantization may vary, but the $L, a, b$ order shown here will prove useful later.

The principal computational advantage of SSQ is that it may be implemented as a sequence of 1-D lookup table (LUT) operations. Therefore, SSQ yields the performance benefits of VQ, but requires no more computation than conventional scalar quantization. Figure 2 illustrates the structure of this sequential LUT for the three-dimensional input $(L, a, b)$. The first LUT quantizes the $L$ component and returns an index $i$ corresponding to the quantization region $L \in S_{i}$. The second LUT then applies the appropriate scalar quantizer based on this region $S_{i}$ and returns the index $(i, j)$ corresponding to the quantization region $(L, a) \in S_{i, j}$. The third LUT quantizes the component $b$ based on $S_{i, j}$ and yields the quantized color vector $Q[L, a, b]=(q L, q a, q b)$.

\subsection{Color Palette Design with SSQ}

To obtain $p_{L a b}(L, a, b)$, we assume that image colors are uniformly distributed over the gamut of the monitor. Define the window function

$w(L, a, b)=\left\{\begin{array}{ll}1 & \text { if }(L, a, b) \text { is in gamut } \\ 0 & \text { if }(L, a, b) \text { is out of gamut }\end{array}\right.$.

Then $p_{L a b}(L, a, b)$ is given by

$p(L, a, b)=\frac{w(L, a, b)}{\int_{L \in M_{i}} \int_{a \in M_{i}} \int_{b \in M_{i}} w(L, a, b) \mathrm{d} L \mathrm{~d} a \mathrm{~d} b}$.

For the time being, we also assume that $N_{1}, N_{2}$, and $N_{3}$ are known in advance. Generally, the total number of desired quantization levels, $N_{3}$, is specified, and we present a method for estimating $N_{1}$ and $N_{2}$ in Sec. 2.2.4.

Our method for designing the SSQ color palette is based on asymptotic quantization theory, ${ }^{18}$ and closely parallels the method used for color quantization in Refs. 4, 11, and 12. One unique aspect of our problem is that we would like to preserve the maximum gamut of the display device. Because halftoning algorithms can produce the average of several palette colors, the effective gamut will be the convex hull spanned by the color palette. Moreover, error diffusion as-

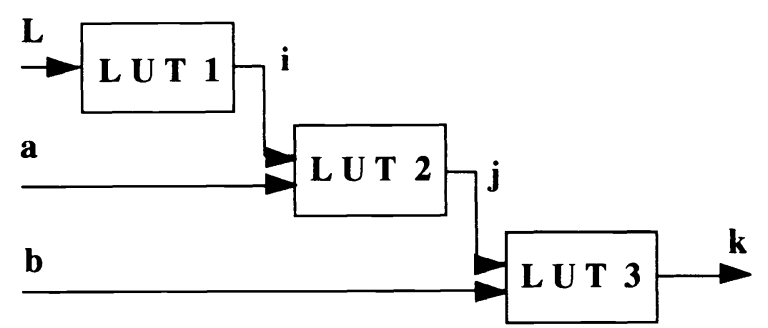

Fig. 2 Block diagram of sequential LUT that performs SSQ quantization for the three-dimensional $(L, a, b)$ input vector. The order of quantization is $L$, then $a$, then $b$, and the output codeword is $Q[L, a, b]$. 
sumes that the input signal is contained in the convex hull of the available quantization levels and will otherwise pass accumulating errors forward to unquantized pixels, thus driving the quantizer into saturation. Therefore, our color palette design method will include heuristics to ensure that colors extend to the boundaries of the device's gamut.

\subsubsection{Luminance quantizer}

First, we design the quantizer for $L$. To preserve the maximum gamut, we temporarily fix the first and last quantization levels to be

$$
\begin{gathered}
\tilde{q}_{1}=\min _{L}\left\{L: p_{L}(L) \neq 0\right\} \\
\tilde{q}_{N_{1}}=\max _{L}\left\{L: p_{L}(L) \neq 0\right\} .
\end{gathered}
$$

Because the computational cost of the quantizer design is not significant, we use the Lloyd-Max algorithm to choose the quantization regions $S_{1}, \ldots, S_{N_{1}}$ and the quantization levels $q_{2}, \ldots, q_{N_{1}-1}$. In practice, we found the performance of the Lloyd-Max algorithm to be strongly dependent on the initial condition. Therefore, we initialize the Lloyd-Max algorithm by choosing the intermediate quantization levels according to the asymptotically optimal point density function $\lambda(L)$, given by

$\lambda(L)=\frac{\left\{p_{L}(L)\right\}^{1 / 3}}{\int_{L \in W_{i}}\left\{p_{L}(x)\right\}^{1 / 3} \mathrm{~d} x}$.

More specifically, we choose the initial values for $q_{2}, \ldots$, $q_{N_{1}-1}$, so that

$\frac{i-1}{N_{1}-1}=\int_{-\infty}^{q_{i}} \lambda(L) \mathrm{d} L$

Figure 3 illustrates a final step in which the quantization levels $\tilde{q}_{1}$ and $\tilde{q}_{N_{1}}$ are replaced with the centroids of their respective quantization regions,

$$
\begin{gathered}
q_{1}=\int_{L \in S_{1}} p_{L}(L) \mathrm{d} L \\
q_{N_{1}}=\int_{L \in S_{N_{1}}} p_{L}(L) \mathrm{d} L .
\end{gathered}
$$

These values of $q_{1}, \ldots, q_{N_{1}}$ are then used as output values for $L$.

\subsubsection{Chrominance quantizer}

The next step is to design the quantizer for the scalar $a$. We first calculate the conditional probability density of $a$ given that $L \in S_{i}$,

$p_{a}\left(a \mid L \in S_{i}\right)=\int_{b \in M i} \int_{L \in S_{i}} p_{L a b}(L, a, b) \mathrm{d} L \mathrm{~d} b$.
Before we can design an optimal quantizer for each region $S_{i}$, we must determine the optimal number of quantization levels $n_{i}$. The optimal value for $n_{i}$ can be derived using asymptotic quantization theory, ${ }^{11}$ and is given by

$n_{i}=\operatorname{int}\left(N_{2} \frac{r_{i}}{\sum_{l=0}^{N_{1}-1} r_{l}}\right)$,

where

$r_{i}=\left\{P\left(L \in S_{i}\right)\right\}^{1 / 3} \int_{a \in M_{i}}\left\{p_{a}\left(a \mid L \in S_{i}\right)\right\}^{1 / 3} \mathrm{~d} a$

$P\left(L \in S_{i}\right)$ is the probability that $L$ is quantized to the region $S_{i}$, and int (.) denotes rounding to the nearest integer.

For each region $S_{i}$ and the bit allocation $n_{i}$, the quantizer for $a$ is designed as before. The Lloyd-Max algorithm is applied after fixing the first and last quantization levels to be

$\tilde{q}_{i, 1}=\min _{a}\left\{a: p_{L a}\left(L=q_{i}, a\right) \neq 0\right\}$

$\tilde{q}_{i, n_{i}}=\max _{a}\left\{a: p_{L a}\left(L=q_{i}, a\right) \neq 0\right\}$,

and the Lloyd-Max algorithm is initialized using

$\frac{j-1}{n_{i}-1}=\int_{-\infty}^{q_{i, j}} \lambda_{i}(a) \mathrm{d} a$,

where

$\lambda_{i}(a)=\frac{\left\{p_{a}\left(a \mid L \in S_{i}\right)\right\}^{1 / 3}}{\int_{x \in M_{i}}\left\{p_{a}\left(x \mid L \in S_{i}\right)\right\}^{1 / 3} \mathrm{~d} x}$.

Once again, as a final step, the first and last quantization levels are relocated to the centroids of their cells according to

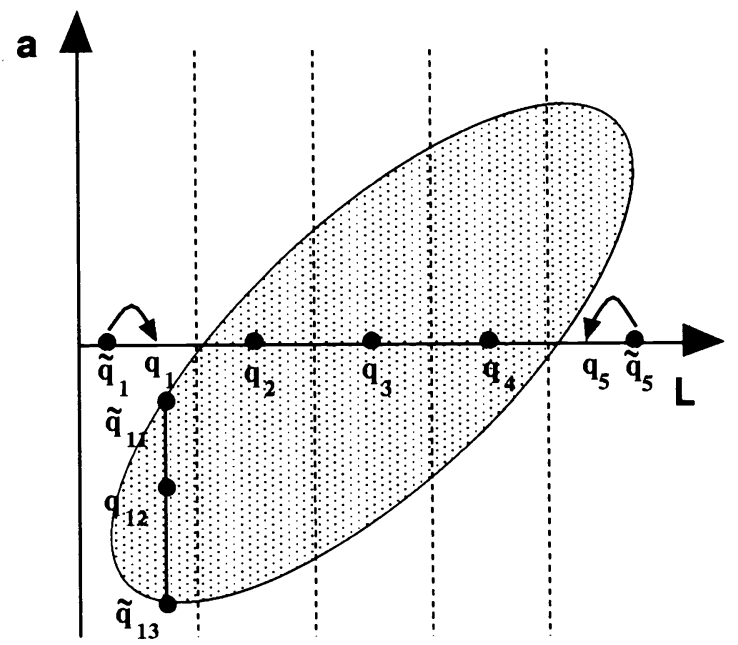

Fig. 3 Quantization procedure for $L$ and a components. Notice that $\tilde{q}_{1}$ and $\tilde{q}_{5}$ are initially placed at the most extreme locations, and are then moved to the centroids of their cells denoted by $q_{1}$ and $q_{5}$. Quantization levels $q_{1, j}$ are lined up along $q_{i}$. 


$$
\begin{gathered}
q_{i, 1}=\int_{L \in S_{i, 1}} p_{a}\left(a \mid L \in S_{i}\right) \mathrm{d} a \\
q_{i, N_{1}}=\int_{L \in S_{i, n_{i}}} p_{a}\left(a \mid L \in S_{i}\right) \mathrm{d} a .
\end{gathered}
$$

The quantizer design for $b$ is similar to that used for $a$. For each region $S_{i, j}$ in the $(L, a)$ plane, we design a 1-D quantizer for the $b$ component. Using the conditional density $p_{b}\left[b \mid(L, a) \in S_{i, j}\right]$, we may compute the number quantization levels for region $S_{i, j}$ as

$n_{i, j}=\operatorname{int}\left(N_{3} \frac{r_{i, j}}{\sum_{k=1}^{N_{1}} \sum_{l=1}^{n_{k}} r_{k, l}}\right)$,

where

$r_{i, j}=\left\{P\left[(L, a) \in S_{i, j}\right]\right\}^{1 / 3} \int_{b \in M_{i}}\left\{p_{b}\left[b \mid(L, a) \in S_{i, j}\right]\right\}^{1 / 3} \mathrm{~d} b$,

and $P\left[(L, a) \in S_{i, j}\right]$ is the probability that $(L, a)$ is quantized to the region $S_{i, j}$.

For each region $S_{i, j}$, we again set the first and last quantization levels to their most extreme values,

$$
\begin{aligned}
q_{i, j, 1} & =\min _{b}\left\{b: p_{L a b}\left(L=q_{i}, a=q_{i, j}, b\right) \neq 0\right\} \\
q_{i, j, n_{i, j}} & =\max _{b}\left\{b: p_{L a b}\left(L=q_{i}, a=q_{i, j}, b\right) \neq 0\right\} .
\end{aligned}
$$

The remaining quantization levels $q_{i, j, 2}, \ldots, q_{i, j, n_{i, j-1}}$ are again allocated using the Lloyd-Max algorithm and an initial condition based on the optimal point density function

$$
\lambda_{i, j}(b)=\frac{\left\{p_{b}\left[b \mid(L, a) \in S_{i, j}\right]\right\}^{1 / 3}}{\int_{x \in W_{i}}\left\{p_{b}\left[b \mid(L, a) \in S_{i, j}\right]\right\}^{1 / 3} \mathrm{~d} x} .
$$

This time we do not replace the first and last quantization levels. Instead, we leave them at the boundary of the color space to preserve the maximum gamut.

At this point, we make some observations about the palette. If the gamut of the device is convex, then the palette colors will fall inside the gamut. However, the quantization levels corresponding to the minimum and maximum values of $a$ will generally not be on the boundary of the gamut. In the next section, we will describe how these colors can be moved slightly to expand the usable device gamut. Note that because the values of $n_{i}$ and $n_{i, j}$ must be rounded to the nearest integer, the total number of allocated colors may not always be equal to the desired numbers of colors. The desired number of colors may be obtained by adding or subtracting single quantization levels according to a mean-squared-error criterion as described in Ref. 11. However, for our application, we simply accept a slightly smaller palette.

\subsubsection{Expanding the color map range}

To maximize the displayable gamut formed by the convex hull of palette colors, we will move some of the colors near the minimum and maximum values of $a$. Figure 4 illustrates how colors corresponding to the regions $S_{i, 1}$ and $S_{i, n_{i}}$ can be moved to expand the gamut.

More formally, for $1<j<n_{i}$, we define the final $(L, a, b)$ palette colors to be

$C_{i, j, k}=\left(q_{i,} q_{i, j}, q_{i, j, k}\right)$,

and for $j=1$ or $n_{i}$, we define

$C_{i, 1, k}=\left(q_{i}, \min _{i, k}, q_{i, 1, k}\right)$

$C_{i, n_{i, k}},=\left(q_{i}, \max _{i, k}, q_{i, n_{i, k}}\right)$

where

$\min _{i, k}=\min _{a}\left\{a: p_{L a b}\left(L=q_{i} ; a, b=q_{i, 1, k}\right)>0\right\}$

$\max _{i, k}=\max _{a}\left\{a: p_{L a b}\left(L=q_{i} ; a, b=q_{i, n_{i, k}}\right)>0\right\}$.

Finally, we expand the color map to the maximum range along the $L$ axis by adding one quantization level for perfect black $(0,0,0)$ and another for perfect white $(100,0,0)$.

As mentioned previously, we note that in each cell $S_{i, j}$ the algorithm already placed the first and last quantization level of $b$ on the boundaries of the display gamut.

\subsubsection{Optimal bit allocation}

A method must still be given for selecting the optimal values of $N_{1}$ and $N_{2}$. If $N_{1}, N_{2}$, and $N_{3}$ are large numbers, then asymptotic quantization theory may be applied. Specifically, if $D_{L}, D_{a}$, and $D_{b}$ are the mean squared error in the $L, a, b$, components, respectively, then the total distortion is given by $D=D_{L}+D_{a}+D_{b}$. Furthermore, each component distortion is approximately equal to

$D_{L} \approx \frac{1}{N_{1}^{2}} \alpha, D_{a} \approx \frac{N_{1}^{2}}{N_{2}^{2}} \beta, D_{b} \approx \frac{N_{2}^{2}}{N_{3}^{2}} \gamma$,

where $\alpha, \beta$, and $\gamma$ are constants that depend on the probability density of the input signal. ${ }^{4,11,12}$ If $\alpha, \beta$, and $\gamma$ are known, then the optimal values for $N_{1}$ and $N_{2}$ are given by

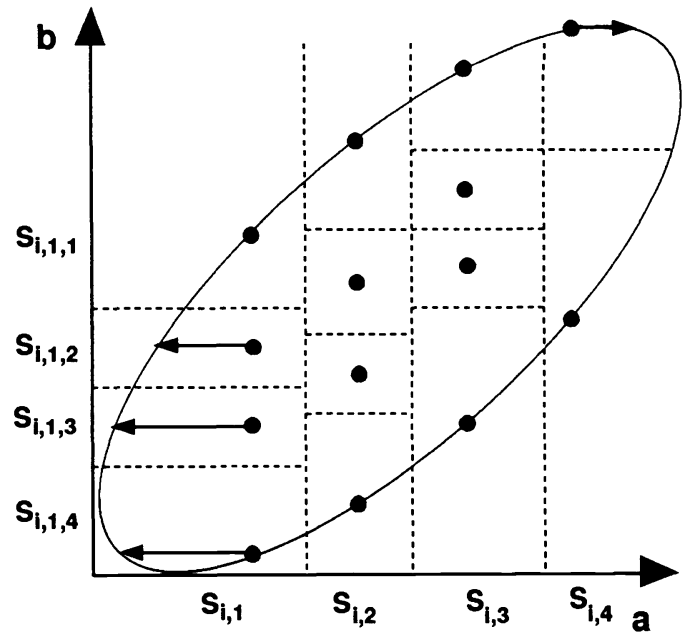

Fig. 4 Expanding the palette range by moving the outer a-quantization levels to the edges of the display gamut. 
$N_{1}=N_{3}^{1 / 3}\left(\frac{(w \alpha)^{2}}{\beta \gamma}\right)^{1 / 6}$

and

$N_{2}=N_{3}^{2 / 3}\left(\frac{w \alpha \beta}{\gamma^{2}}\right)^{1 / 6}$

In practice, $N_{1}, N_{2}$, and $N_{3}$ will not be very large, so the analytical formulas for $\alpha, \beta$, and $\gamma$ will not be accurate. However, experiments have shown that, if the constants $\alpha$, $\beta$, and $\gamma$ are properly adjusted, then Eq. (4) holds for a range of relatively small $N_{k}$. Adjusted values of these constants may be obtained by performing an initial quantization with arbitrary $\tilde{N}_{k}$ and measuring the values of $D_{L}, D_{a}$, and $D_{b}$. The estimates for $\alpha, \beta$, and $\gamma$ may then be obtained by equating terms in Eq. (4).

\section{Application to Error Diffusion}

In this section, we incorporate the optimized color palette into the error diffusion halftoning method. A naive implementation would require a transformation both to and from the $L a b$ coordinate system used in the palette design. However, we show that by careful selection of the coordinates used for the error diffusion algorithm, the SSQ quantizer may be implemented directly without any transformations.

Figure 5 illustrates the basic error diffusion algorithm as described by Floyd and Steinberg in Ref. 7. To better understand how error diffusion works, we review the frequency analysis of Ref. 3. The error diffusion algorithm works by feeding back quantization error to reduce the low-frequency component of the displayed error. Let $s(n)$ be an image indexed by the two-dimensional pixel locations $n=\left(n_{1}, n_{2}\right)$. Then, the equations that describe error diffusion are given by

$$
\begin{aligned}
& y(n)=Q[\tilde{s}(n)] \\
& q(n)=\tilde{s}(n)-y(n) \\
& \tilde{s}(n)=s(n)+g(n) * q(n),
\end{aligned}
$$

where $y(n)$ is the displayed image, $q(n)$ is the quantization error, and $*$ denotes two-dimensional convolution. Substituting the third equation into the second yields the relationship for the display error $e(n)$,

$$
\begin{aligned}
e(n) & =s(n)-y(n) \\
& =q(n)-g(n) * q(n) .
\end{aligned}
$$

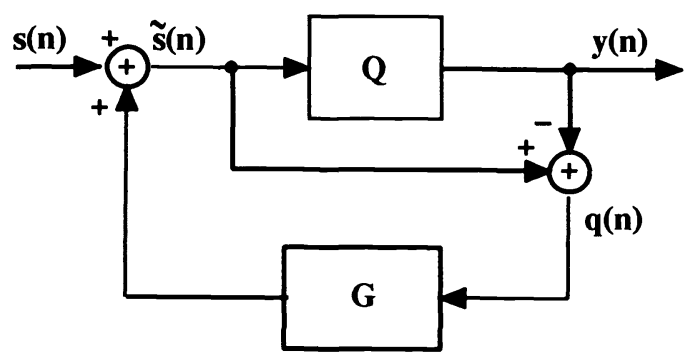

Fig. 5 Block diagram of the error diffusion algorithm.
Because this is a linear relationship, we may take the frequency transform to yield

$E(\omega)=[1-G(\omega)] Q(\omega)$,

where $E(\omega), G(\omega)$, and $Q(\omega)$ denote the discrete space Fourier transforms of $e(n), g(n)$, and $q(n)$, respectively. Thus, the display error spectrum can be shaped by selecting the proper error diffusion filter $G(\omega)$. Generally, $G(\omega)$ is chosen to be lowpass so that the transfer function $1-G(\omega)$ is highpass. This suppresses the low-frequency error for which the visual system is most sensitive.

We choose the error diffusion filter $G(\omega)$ to minimize the perceived error as described in Ref. 13. This optimized error diffusion approach is appropriate for multilevel halftoning and selects $G(\omega)$ based on an overall system model. This system model includes the monitor and the human visual system response, and incorporates the contrast sensitivity to both luminance and chrominance, and the reduced visual sensitivity to diagonal frequencies.

One difficulty with incorporating the optimized palette into error diffusion is the proper choice of color coordinates. For the perceived color of the original and halftone images to match, the data should be processed in the same coordinate system used by the human visual system. Experiments show that spatial frequency response of the visual system is due to the combined effects of optical blur and the limited resolving power of the retina-brain system. ${ }^{19}$ Recent studies have found that the falloff in contrast sensitivity at high spatial frequencies is mainly due to optical properties of the eye. ${ }^{20}$ Because optical blurring effects are due to incoherent averaging of energy, these effects are properly modeled by filtering in a color coordinate system that is linear in intensity. In our application, this lowpass behavior is the dominant effect, so we adopt a linear color coordinate system when modeling the lowpass nature of the human visual system. (It is interesting to note that conventional color measurement instruments average the energy of the reflected signal over a color patch, and therefore effectively operate in a linear color coordinate system.) This choice precludes the direct use of the $L a b$ coordinates in the error diffusion algorithm because they are nonlinearly related to intensity.

Figure 6(a) shows a naive solution to this problem of mismatched coordinate systems. The input data $s(n)$ is processed by the error diffusion filter in a linear coordinate system. However, because the quantizer is designed in $L a b, T$ $(\cdot)$ transforms the colors to $L a b$ before quantization and $T^{-1}(\cdot)$ converts back after quantization. We would like to eliminate these transformations because they are computationally expensive. The inverse transformation $T^{-1}(\cdot)$ may be eliminated by precomputing the transformation for the palette of output colors.

We will eliminate the forward transformation $T(\cdot)$ by judiciously selecting the linear coordinate system for error diffusion. Define the coordinate system ${ }^{14}$ that we call $Y_{y}$, $c_{x}, c_{z}$

$Y_{y}=116 \frac{Y}{Y_{n}}$

$c_{x}=500\left[\frac{X}{X_{n}}-\frac{Y}{Y_{n}}\right]$, 


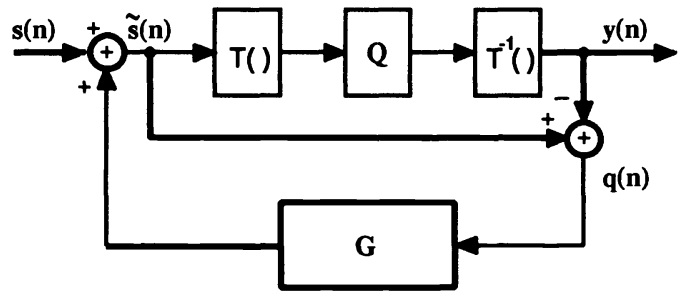

(a)

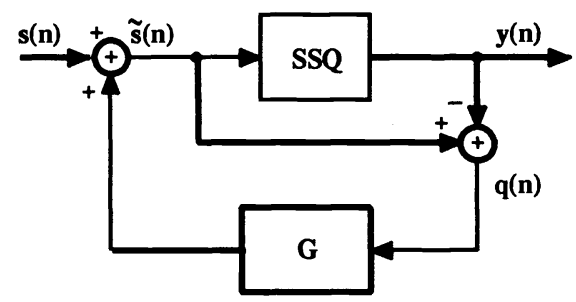

(b)

Fig. 6 Block diagram of error diffusion with SSQ: (a) matching color spaces with transformations $T$ and $T^{-1}$ and (b) eliminating transformations in new $Y_{y}, c_{x}, c_{z}$ color space.

$c_{z}=200\left[\frac{Y}{Y_{n}}-\frac{Z}{Z_{n}}\right]$

where as before $\left(X_{n}, Y_{n}, Z_{n}\right)$ specifies the white point. Note that the $Y_{y} c_{x} c_{z}$ coordinates are chosen so that they are aligned with the $L, a, b$ system. In fact, one may easily verify that

$\left.\nabla_{\left(Y_{y}, c_{x}, c_{z}\right)}(L, a, b)\right|_{\text {white point }}=f^{\prime}\left(Y_{y}\right) \mid$

where $f^{\prime}\left(Y_{y}\right)$ is the derivative of $f(\cdot)$ evaluated at $Y_{y}$ and $\mathbf{I}$ is the identity matrix. Furthermore, $Y_{y}, c_{x}, c_{z}$ is approximately an opponent color system, where variations in $c_{x}$ correspond to changes along a red-green direction and variations in $c_{z}$ reflect changes along a blue-yellow axis.

We show next that the SSQ structure of the optimized palette is approximately preserved in the coordinate system $Y_{y}, c_{x}, c_{z}$. This implies that a modified SSQ quantizer may be directly applied to the components of $\tilde{s}(n)$ in the error diffusion filter. Because the function $f(\cdot)$ in Eq. (2) is a monotone increasing function, quantization of $L=116 f\left(Y / Y_{n}\right)-16$ may be replaced by equivalent quantization of $Y_{y}$,

$Q_{L}[L] \equiv Q_{Y}\left[Y_{y}\right]$.

The component $a$ is dependent on the value of $Y_{y}$ and $c_{x}$, however, the value of $Y_{y}$ may be approximated by the quantized value $Q_{Y}\left[Y_{y}\right]$. After making this replacement, the quantization of $a$ is equivalent to quantization of $c_{x}$. More specifically, we may define the function $a=g\left(c_{x}, Y_{y}\right)$. Then

$$
\begin{aligned}
Q_{a}[a] & =Q_{a}\left[g\left(c_{x}, Y_{y}\right)\right] \\
& \approx Q_{a}\left[g\left(c_{x}, Q_{Y}\left[Y_{y}\right]\right)\right] \\
& =Q_{c x}\left[c_{x}, Q_{Y}\left[Y_{y}\right]\right] .
\end{aligned}
$$

Therefore, for each quantized value $Q_{Y}\left[Y_{y}\right]$, the quantizer for $a$ may be approximately replaced by a quantizer for $c_{x}$. We note that this approximation becomes more accurate as $N$ becomes large.

The third component is similar. Define the function $b=h\left(c_{z}, Y_{y}\right)$. Then

$$
\begin{aligned}
Q_{b}[b] & =Q_{b}\left[h\left(c_{z}, Y_{y}\right)\right] \\
& \approx Q_{b}\left[h\left(c_{z}, Q_{Y}\left[Y_{y}\right]\right)\right] \\
& =Q_{c z}\left[c_{z}, Q_{Y}\left[Y_{y}\right]\right] .
\end{aligned}
$$

This implies that the quantizer for $b$ may be replaced by a quantizer for $c_{z}$ for each quantized value $Q_{Y}\left[Y_{y}\right]$.

Figure 6(b) shows a block diagram of the resulting error diffusion algorithm, where we assume that the input image $s(n)$ is already transformed to $Y_{y}, c_{x}, c_{z}$ coordinates. Note that the transformations before and after the quantizer are eliminated and that the block diagram resembles basic error diffusion as in Fig. 2, where the simple RGB quantizer is replaced by a sequential LUT similar to Fig. 2 but with $Y_{y}$, $c_{x}, c_{z}$ inputs.

\section{Visually Weighted Error Metric}

We would like to evaluate the quality of a halftoned image by some reproducible, perceptually relevant criterion. For large color patches, it is common to calculate the mean squared error,

$\Delta E=\Delta L^{2}+\Delta a^{2}+\Delta b^{2}$,

where $\Delta L, \Delta a$, and $\Delta b$ are the differences between the original and the reproduced color patch in the visually uniform color space $L a b$. As mentioned previously, error diffusion and other halftoning techniques introduce mostly highfrequency noise to the quantized image. Therefore, any useful error metric must incorporate a model for human contrast sensitivity as a function of spatial frequency. Mitsa and Varkur ${ }^{21}$ have studied a variety of quality metrics for monochrome halftone images and have found that the best correlation with subjective tests is achieved when the frequency response is chosen to be lowpass instead of bandpass. We take a similar approach, but also incorporate the Lab color metric to account for nonlinear visual effects.

Figure 7 shows a block diagram of our error metric. First, the halftoned and the original image are transformed from RGB to $Y_{y}, c_{x}, c_{z}$ coordinates. Both images are then passed

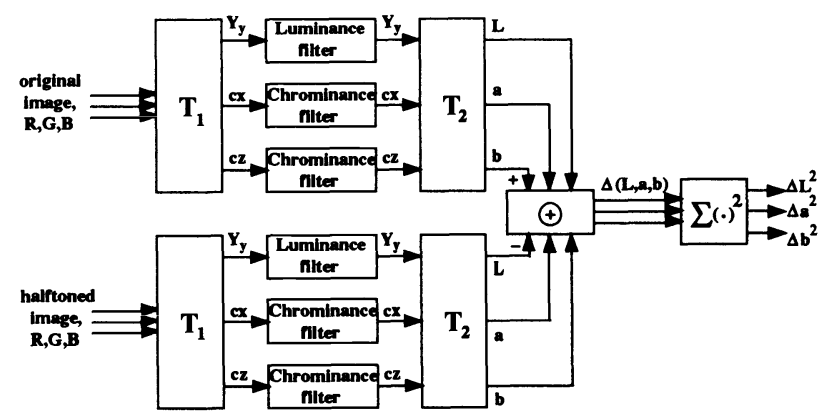

Fig. 7 Block diagram of the visually weighted error metric: $T_{1}, T_{2}$ denote the transformations from R, G,B to $Y_{y}, c_{x}, c_{z}$ and from $Y_{y}, c_{x}$, $c_{z}$ to $L, a, b$. 
through a set of lowpass modulation transfer functions, which model the contrast sensitivity of the human observer toward luminance and chrominance. The outputs of these filters are converted to $L a b$ coordinates and the difference signals between the halftone and original are calculated. Finally, the energy in each component of the difference signal is determined. Before combining the three values of $\Delta L^{2}, \Delta a^{2}$, and $\Delta b^{2}$ to a $\Delta E$ related quantity, we examine the performance of our algorithm in the three components $L, a, b$ separately.

Note that the linear filtering operation is performed before the transformation to the $L a b$ coordinates. This order of operations is critical, because the perceived color will be the average of the halftoned colors in a linear coordinate system. If the nonlinear transformation were performed first, the model would inaccurately predict the perceived color of the halftoned image.

The spatial filters used in luminance and chrominance are based on visual model described by Sullivan, Ray, Miller, and Pios ${ }^{22,23}$ and data obtained by Mullen. ${ }^{24}$ Both the luminance and the chrominance model are of the form

$W(\tilde{f})=\left\{\begin{array}{ll}\exp \left\{-\alpha\left(\tilde{f}-f_{c}\right)\right\} & f \geqslant f_{c} \\ 1 & f<f_{c}\end{array}\right.$,

where the decay rates $\alpha$ and the cutoffs $f_{c}$ are estimated from Mullen's data. For the luminance model, we determined $\alpha=0.4385 \mathrm{deg} /$ cycle and $f_{c}=2.2610$ cycles $/ \mathrm{deg}$, and for the chrominance model we obtained $\alpha=0.1761 \mathrm{deg} /$ cycle and $f_{c}=0.2048$ cycles $/ \mathrm{deg}$. Furthermore, $\tilde{f}$ is the weighted magnitude of the frequency vector $f=\left(f_{1}, f_{2}\right)$, where the weighting has an angular dependence as applied by Sullivan, ${ }^{22}$

$\tilde{f}=\frac{\left(f_{1}^{2}+f_{2}^{2}\right)^{1 / 2}}{s(\Theta)}$,

where

$s(\Theta)=0.15 \cos (4 \Theta)+0.85$,

and $\Theta$ is defined as

$\Theta=\arctan \left(\frac{f_{1}}{f_{2}}\right)$.

Thus, the model is also a function of the viewing angle and decreases faster for diagonal frequencies to account for reduced sensitivity to luminance changes in diagonal directions.

\section{Experimental Results}

The marginal distributions are obtained by numerical integration of $p_{L a b}(L, a, b)$ with respect to $a$ and $b$. Figure 8 shows a histogram of this color distribution in the $(L, a)$ plane for a display with SMPTE RGB primaries and a D65 white point. Bright areas correspond to high probability, while the dark regions along the boundaries of the gamut reflect low probability. The white dots in Fig. 8 indicate the positions of the quantization levels projected into the $(L, a)$ plane and the white lines show the boundaries between quantization cells. Note that several quantization levels are shown for most of the boundary quantization cells. This shows the effect of

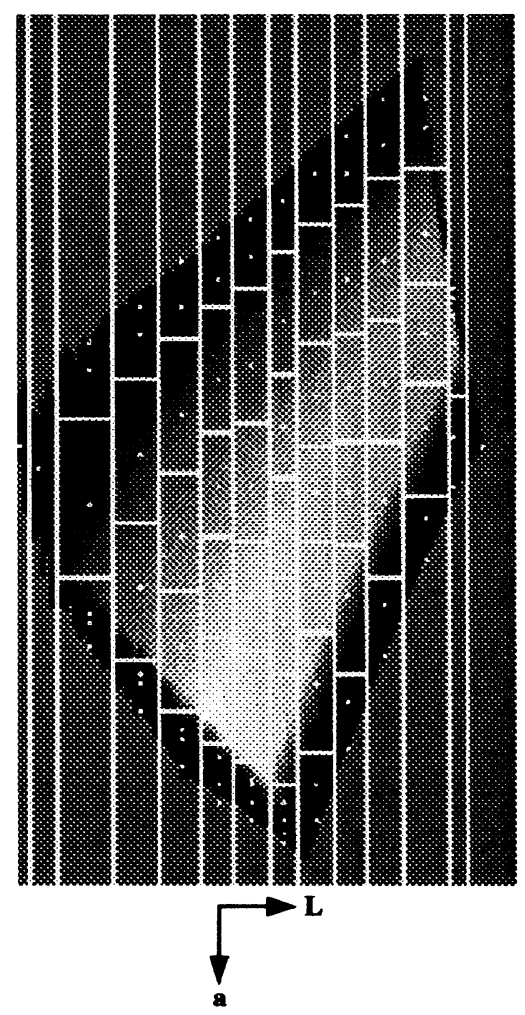

Fig. 8 Histogram of the marginal color distribution in the $(L, a)$ plane, where $L$ increases from left to right and $a$ increases from top to bottom. Bright areas indicate high probability and dark regions reflect low probability.

expanding the range of the color map as described in Sec. 2.2.3. Using a weighting factor of $w=8.0$ for the error metric, we assign 12 quantization levels to luminance, 57 levels to the chrominance component $a$, and 245 colors to the entire palette. Adding perfect black and perfect white to the palette increases the palette size to 247 colors.

Figure 9 illustrates the transformation of the color map and the histogram to the new $Y_{y}, c_{x}, c_{z}$ coordinate system, where the $Y_{y}, c_{x}$ plane is shown. Note that the sequential structure of the color palette is maintained after the transformation.

Figure 10 shows the range of colors in the $(L, a)$ plane, which occur in the image "Picnic." Light gray areas indicate colors that are present in the image. The white lines and dots again indicate the quantization cell boundaries and the palette colors. For each quantization region, we only show the one palette color that is closest to the gamut boundary. Note how the colors of this image extend almost to the edges of the projected gamut. Although the distribution of colors in any one image is rarely uniform, the figure shows the variety of colors that may occur in one image. This indicates the value of minimizing the loss in gamut when designing the color map.

We compare three algorithms: Floyd-Steinberg with a separable palette, Floyd-Steinberg with an optimized palette, and optimized error diffusion with an optimized palette. We refer to this last combination as the optimized algorithm. Because a separable RGB color palette with linear spacings between quantization levels yields poor results, we used a separable 


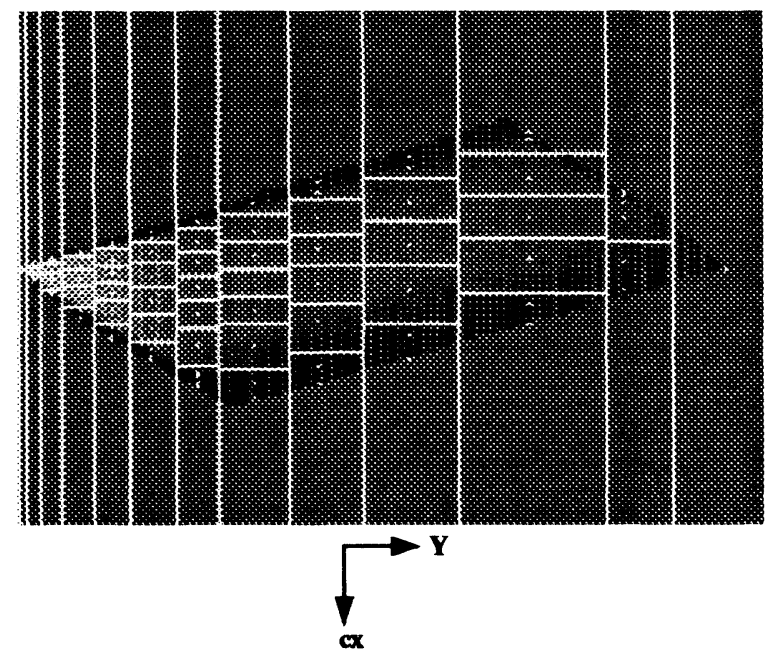

Fig. 9 Histogram of the marginal color distribution in the $\left(Y_{y}, C_{x}\right)$ plane, where $Y_{y}$ increases from left to right and $c_{x}$ increases from top to bottom. The $Y_{y}$ axis is magnified by a factor of 8 .

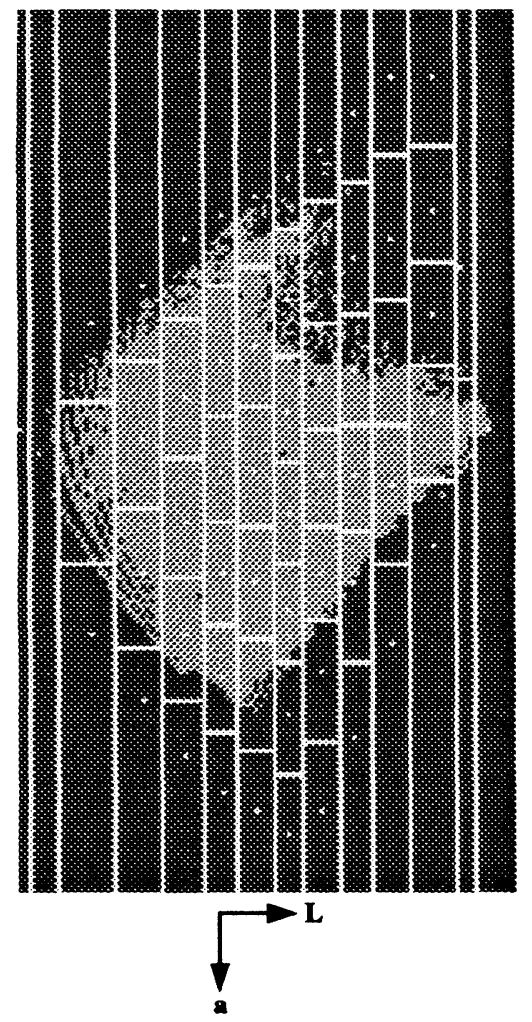

Fig. 10 Range of color distribution in the $(L, a)$ plane for the image "Picnic."

RGB palette with colors allocated according to a power law with an exponent of 3.0. A similar approach was taken by Goertzel and Thompson in Ref. 9. We assigned eight levels to each of the red and green components, and four levels to the blue component for a total of 256 colors.

Table 1 shows a measure of perceived error for a set of five test images shown in Fig. 11. The error was measured using the procedure described in Sec. 4 and assumed a normal
Table 1 Estimates of perceived error of halftone images in $L, a, b$ The optimized error diffusion algorithm with an optimized universa palette produced the lowest errors in almost every case.

\begin{tabular}{|l|c|l|l|l|}
\hline Image: & & $\begin{array}{l}\text { Floyd-Steinberg } \\
\text { RGB Palette }\end{array}$ & $\begin{array}{l}\text { Floyd-Steinberg } \\
\text { Opt. Palette }\end{array}$ & $\begin{array}{l}\text { Opt. ED } \\
\text { Opt. Palette }\end{array}$ \\
\hline Balloon & $\Delta L^{2}$ & 0.3176 & 0.1239 & 0.0872 \\
& $\Delta a^{2}$ & 0.1203 & 0.0772 & 0.0510 \\
& $\Delta b^{2}$ & 0.7894 & 0.3310 & 0.2940 \\
\hline Beach & $\Delta L^{2}$ & 0.3584 & 0.1191 & 0.0740 \\
& $\Delta a^{2}$ & 0.1064 & 0.0238 & 0.0116 \\
& $\Delta b^{2}$ & 0.3889 & 0.0524 & 0.0357 \\
\hline Munich & $\Delta L^{2}$ & 0.3190 & 0.1356 & 0.0968 \\
& $\Delta a^{2}$ & 0.0662 & 0.0428 & 0.0191 \\
& $\Delta b^{2}$ & 0.1092 & 0.0300 & 0.0168 \\
\hline Picnic & $\Delta L^{2}$ & 0.4016 & 0.2680 & 0.1989 \\
& $\Delta a^{2}$ & 0.1675 & 0.1726 & 0.1477 \\
& $\Delta b^{2}$ & 0.4257 & 0.4031 & 0.4514 \\
\hline Uchart & $\Delta L^{2}$ & 0.3298 & 0.2037 & 0.1535 \\
& $\Delta a^{2}$ & 0.1247 & 0.0751 & 0.0355 \\
& $\Delta b^{2}$ & 0.6661 & 0.3668 & 0.2614 \\
\hline
\end{tabular}

viewing distance of $45.5 \mathrm{~cm}$ (17.9 in.) and a display resolution of 100 dots/in. We examined a variety of images, including images with very saturated colors such as "Picnic," images with more pastel colors such as "Beach" and "Balloon," and test images such as "Uchart." Except for the $b$ component in the image "Picnic," the optimized algorithm achieved consistently lower errors in all three components. Column 2 in Table 1 shows the improvement that can be achieved by using the optimized color palette while retaining the Floyd-Steinberg filter coefficients. However, the best overall performance was achieved when combining the optimized color palette with optimized filter coefficients. These numerical results corresponded well with our subjective evaluations of image quality. Table 2 lists values of $\Delta$ $E$ for the three algorithms, which are obtained by adding the three quantities of $\Delta L^{2}, \Delta a^{2}, \Delta b^{2}$ for each image. In this case, the optimized error diffusion together with an optimized color palette consistently produced the lowest errors.

Figure 12 shows the original "Balloon'" image, the FloydSteinberg halftone with RGB palette, Floyd-Steinberg algorithm with optimized palette, and optimized error diffusion with optimized palette. Given the 2:1 zoom of the figures and the assumed viewing distance and resolution, these images are designed to be viewed at 7.0 times their height. The range of colors in this image and the numerical results for our error metric seem to be typical for the majority of images we worked with. Note that the optimized algorithm breaks up contours and patterns that are typical artifacts of the FloydSteinberg algorithm. These effects can be most clearly seen in the slowly varying colors of the different balloons. Note, for example, how contouring effects in the balloon on the left of the girl's face are broken up when using the fully optimized algorithm.

Figure 13 shows the original "Picnic" image, the FloydSteinberg halftone with RGB palette, Floyd-Steinberg algorithm with optimized palette, and optimized error diffusion with optimized palette. Similar effects may be seen in this image. For instance, examining the texture in the tree on the right side of the image, it can be seen that the optimized algorithm renders details with more accuracy than the old method. However, the improvement in this image was the 


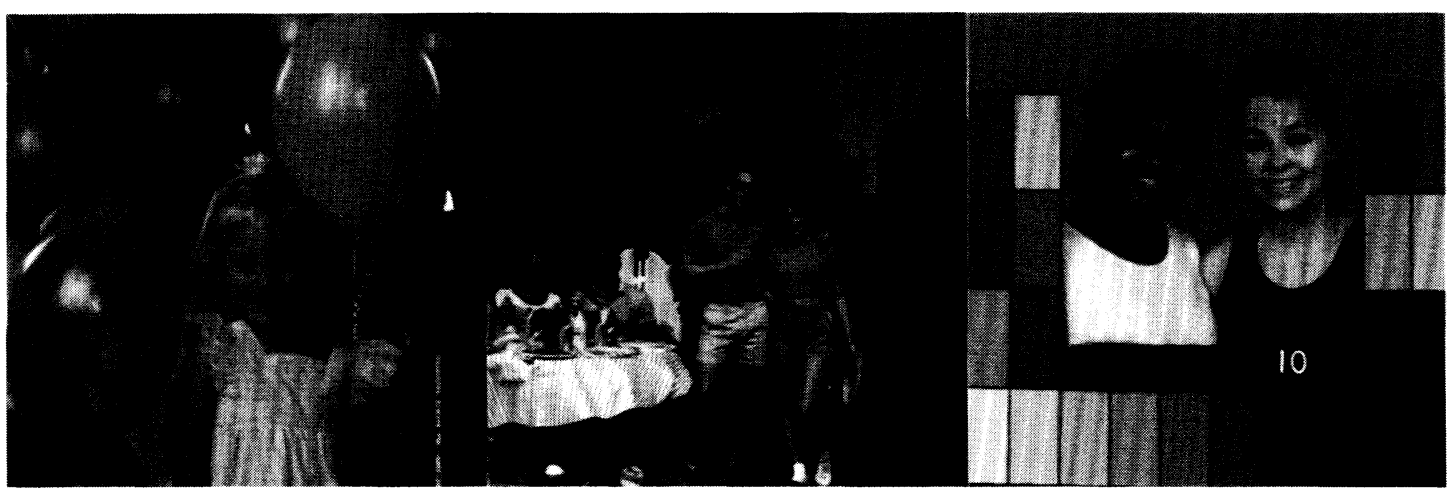

(a)

(b)

(c)

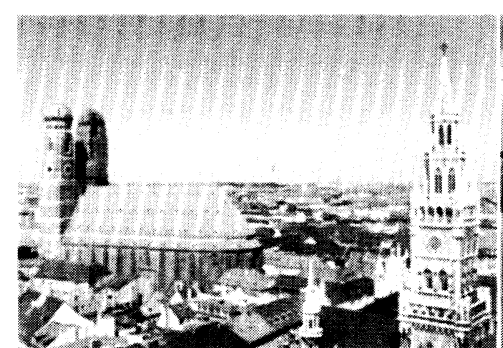

(d)

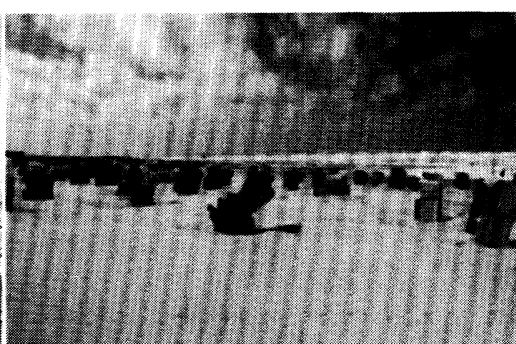

(e)
Fig. 11 Test images: (a) "Balloon," tones obtained from color images.)

Table 2 Estimates of perceived error of haltone images in $\Delta E^{2}$ Here the optimized error diffusion algorithm with an optimized universal palette yielded consistently the lowest errors.

\begin{tabular}{|c|c|c|c|}
\hline Image: & $\begin{array}{c}\text { Floyd-Steinberg } \\
\text { RGB Palette } \\
\Delta E^{2}\end{array}$ & $\begin{array}{c}\text { Floyd-Steinberg } \\
\text { Opt. Palette } \\
\Delta E^{2}\end{array}$ & $\begin{array}{c}\text { Opt. ED } \\
\text { Opt. Palette } \\
\Delta E^{2}\end{array}$ \\
\hline Balloon & 1.2273 & 0.5321 & 0.4322 \\
Beach & 0.8537 & 0.1953 & 0.1213 \\
Munich & 0.4944 & 0.2084 & 0.1327 \\
Picnic & 0.9948 & 0.8437 & 0.7980 \\
Uchart & 1.1206 & 0.6456 & 0.4504 \\
\hline
\end{tabular}

least noticeable and the numerical results represent our worsecase performance.

In a few image areas, for instance in the blue sky area of the "Picnic" image, the optimized algorithm seems to generate slightly more visible texture. We think that this effect is our trade-off for achieving an overall performance improvement. The predominantly diagonal orientation of the texture may be due to the angle dependency of the human visual model, which was used to design our optimized error diffusion filter.

\section{Conclusion}

We examined the design of a universal color palette in a visually uniform color space. The palette is generated using a new vector quantization method known as SSQ. The SSQ approach reduces computation by allowing VQ to be performed with a series of scalar quantizers. The resulting color palette was then combined with a previously developed multilevel error diffusion algorithm to give the best overall result.
To evaluate the quality of the displayed images, we developed a visually weighted error metric that uses models for human contrast sensitivity toward luminance and chrominance together with a nonlinear transformation of the color space. The measurement of this error metric for a variety of images substantiated our subjective conclusions that the optimized palette improved the displayed image quality.

\section{Acknowledgments}

We would like to thank the NEC Corporation for their support of this work. Furthermore, we wish to thank Kodak and Reiner Eschbach from Xerox Corporation for providing some of our test images.

\section{References}

1. P. Heckbert, "Color image quantization for frame buffer display," Comput. Graphics, 16(3), 297-307 (1982).

2. G. Braudaway, "A procedure for optimum choice of a small number of colors from a large color palette for color imaging,' IBM internal report, RC 11367 (\#51227) (Sep. 16, 1985).

3. M. T. Orchard and C. A. Bouman, "Color quantization of images," IEEE Trans.Signal Process. 39(12), 2677-2690 (1991).

4. R. Balasubramanian, C. A. Bouman, and J. P. Allebach, "Sequential scalar quantization of color images,' IS\&T's 46th Annual Conf., pp. 97-101, Boston, MA, (May 9-14, 1993).

5. V. Iverson and E. Riskin, "A fast method for combining palettes of color quantized images," Proc. of IEEE Int'l. Conf. on Acoustic Speech Signal Process., Minneapolis, MN, 5, 317-320 (April 27-30, 1993).

6. R. S. Gentile, E. Walowit, and J. P. Allebach, "Quantization and multilevel halftoning of color images for near original image quality,"' $J$. Opt. Soc. Am. A. 7(6), 1019-1026 (1990).

7. R. W. Floyd and L. Steinberg, "An adaptive algorithm for spatial greyscale," Proc. SID 17(2), 75-77 (1976)

8. R. A. Ulichney, “Dithering with blue noise,"' Proc. IEEE 76(1), 56-79 (1988). 


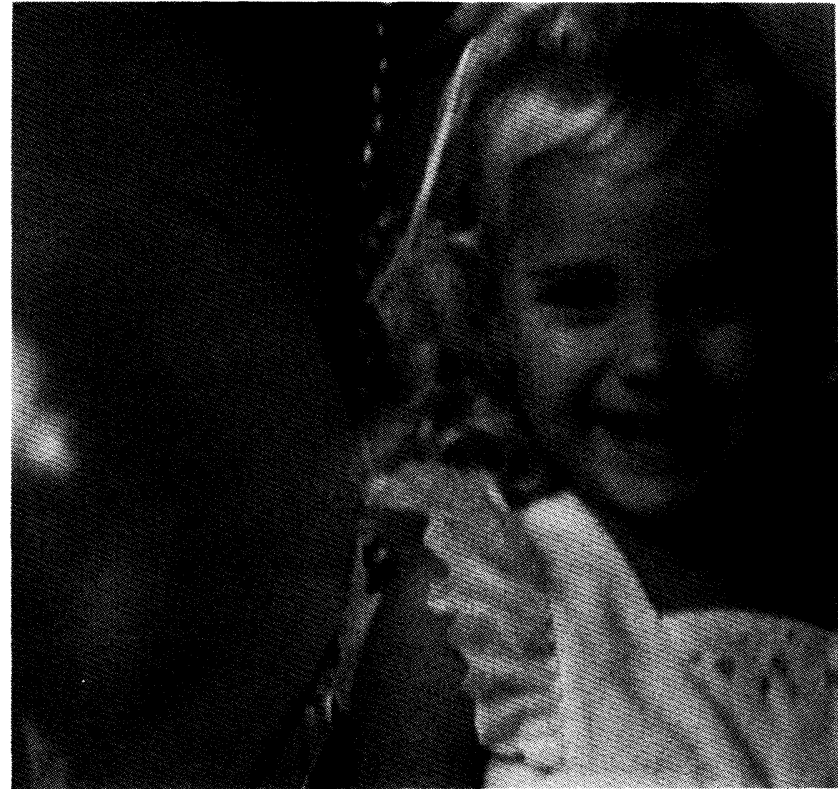

(a)

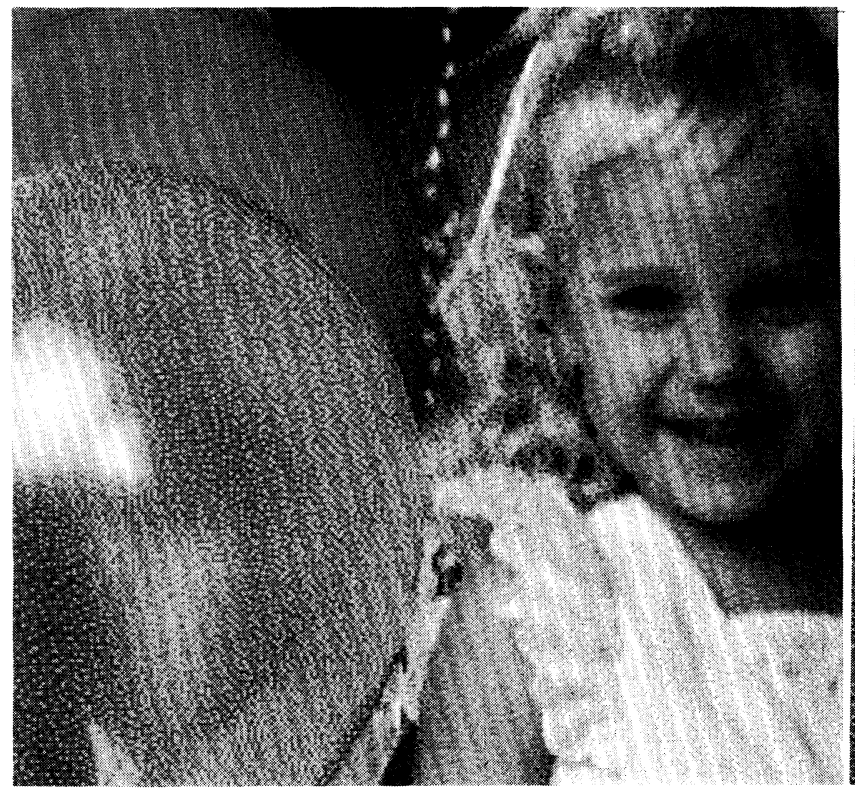

(c)

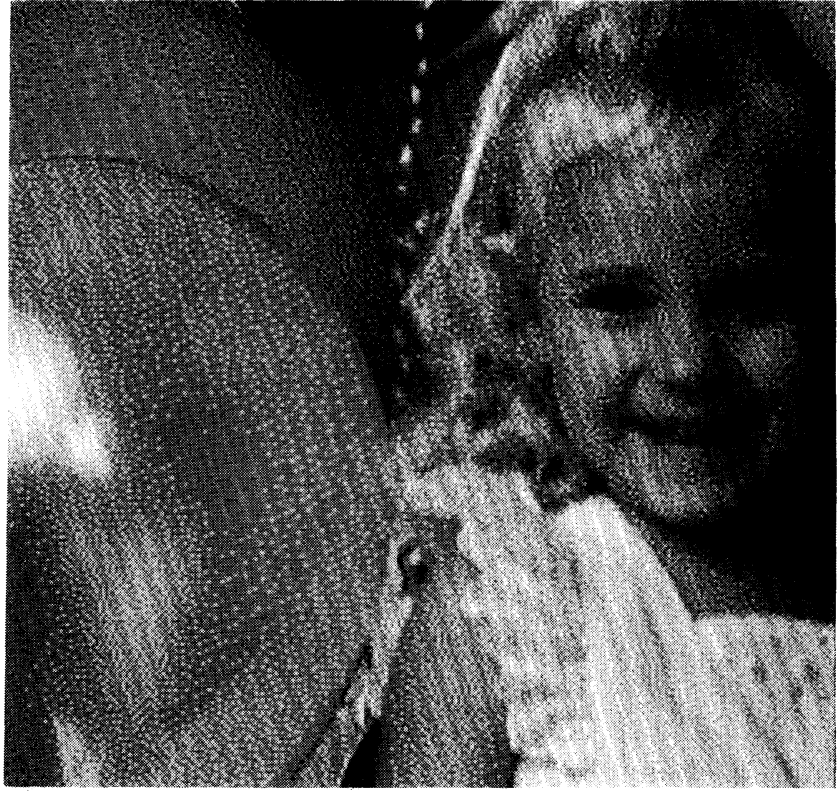

(b)

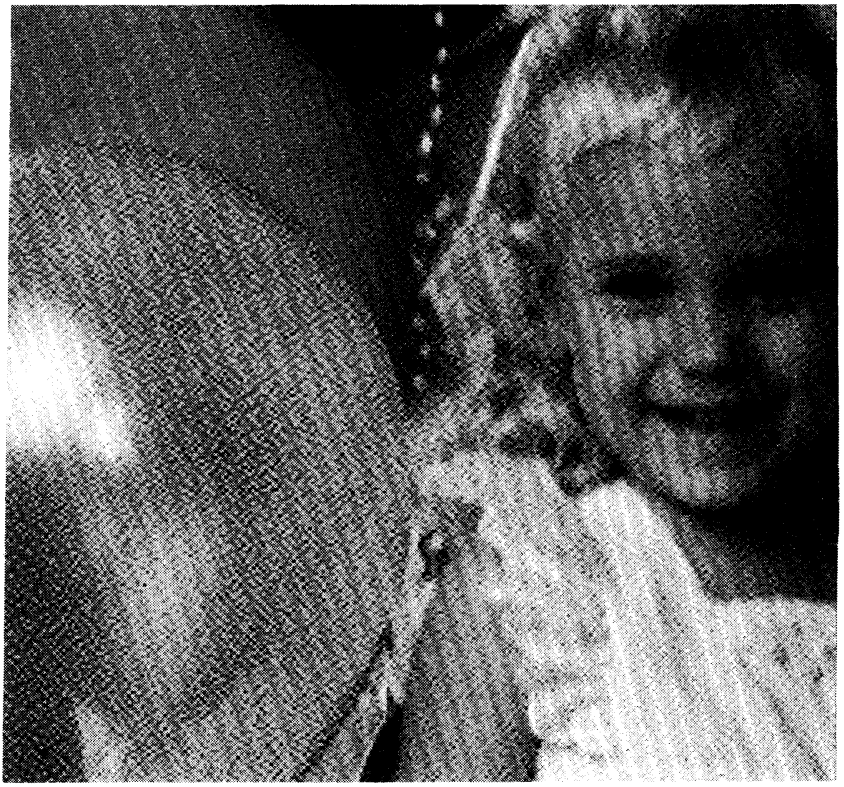

(d)

Fig. 12 Comparison of 2:1 zoom of (a) original "Balloon" image, (b) Floyd-Steinberg algorithm with separable RGB palette, (c) Floyd-Steinberg algorithm with optimized palette, and (d) optimized error diffusion with optimized palette. (Halftones obtained from color images.)

9. G. Goertzel and G. R. Thompson, "'Halftoning' techniques for displaying images with a limited color palette,' EI West 1990, 102-108, Pasadena, CA (1990).

10. D. Venable, J. Stinehour, and P. Roetling, "Selection and use of small color sets for pictorial display,' SPSE 43rd Annual Meeting, 90-92. Rochester, NY (May 20-25, 1990).

11. R. Balasubramanian, C. A. Bouman, and J. P. Allebach, "Sequential scalar quantization of vectors: an analysis," (submitted for publication).

12. R. Balasubramanian, C. A. Bouman, and J. P. Allebach, "Sequential scalar quantization of color images,' J. Electron. Imag., 3(1), 45-59 (1994).

13. B. W. Kolpatzik and C. A. Bouman, "Optimized error diffusion for image display,'” J. Electron. Imag., 1(3), 277-292 (1992).
14. T. J. Flohr, B. W. Kolpatzik, R. Balasubramanian, D. A. Carrara, C. A Bouman, and J. P. Allebach, "Model based color image quantization,' Proc. SPIE/IS\&T Conf. Human Vision, Visual Process., Digital Display, 1993, 270-281, San Jose, CA (Jan. 31-Feb. 4, 1993).

15. J. Makhoul, S. Roucos, and H. Gish, "Vector quantization in speech coding,'” Proc. IEEE, 73(11), 1551-1588 (1985).

16. W. H. Equitz, "A new vector quantization clustering algorithm," IEEE Trans. Acoust., Speech, Signal Process., 37(10), 1568-1575 (1989).

17. R. Balasubramanian and J.P. Allebach. "A new approach to palette selection for color images,' J. Imag. Technol. 17(6), 284-290 (1991).

18. A. Gersho and R. M. Gray, Vector Quantization and Signal Compression, Kluwer Academic Publishers, Norwell, MA (1992).

19. F. W. Campbell, "The human eye as an optical filter," Proc. IEEE 


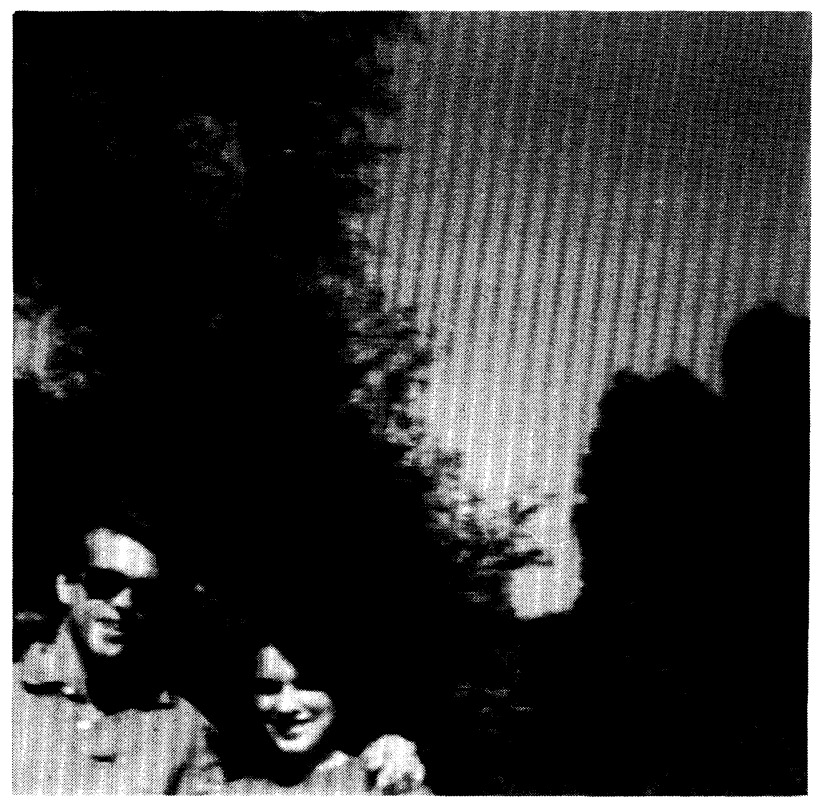

(a)

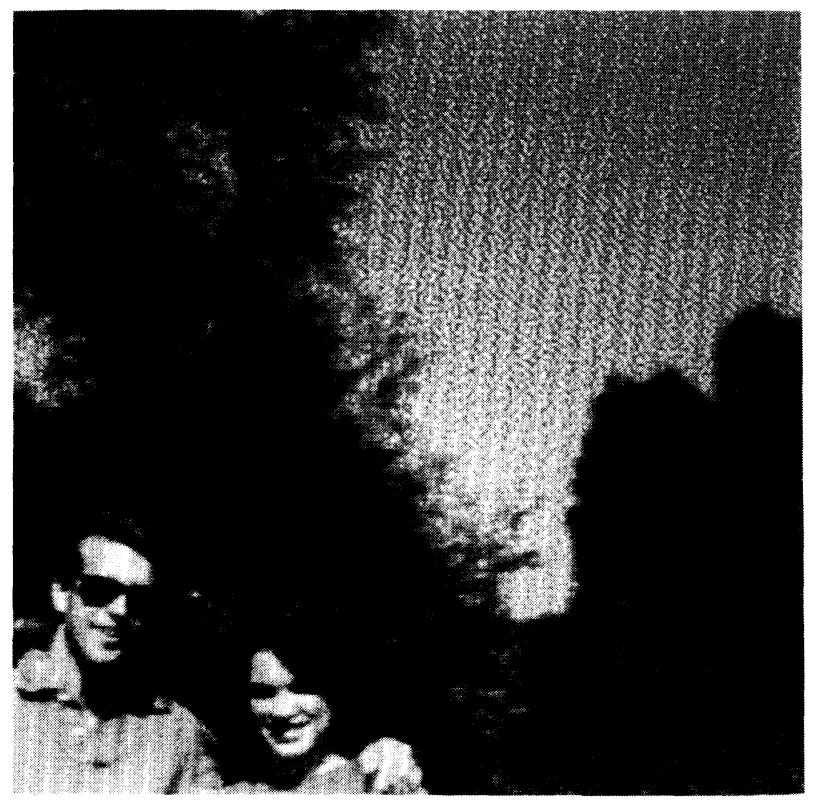

(c)

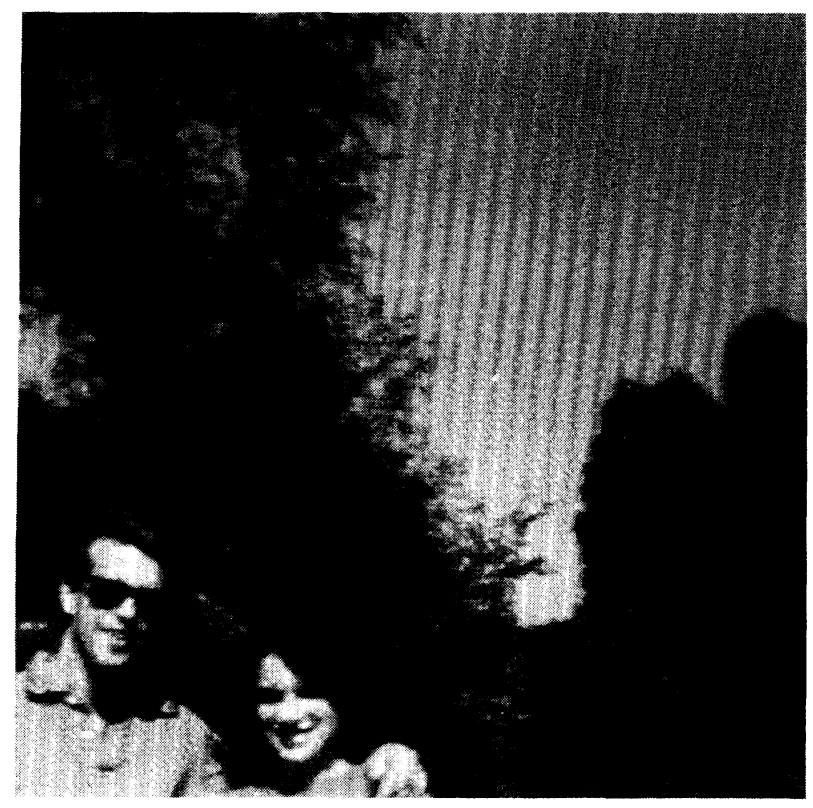

(b)

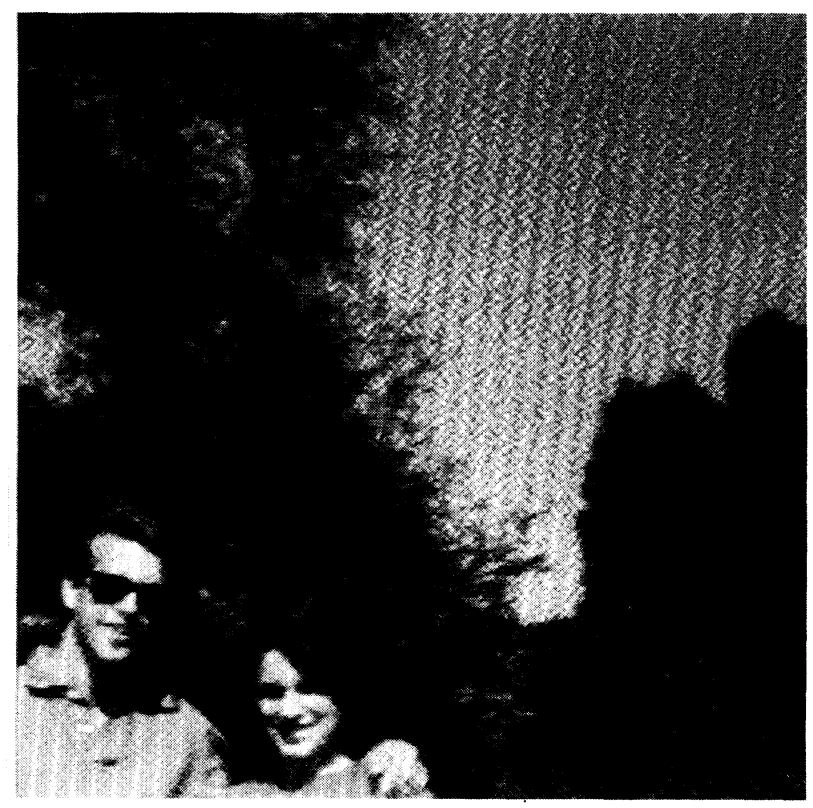

(d)

Fig. 13 Comparison of 2:1 zoom of (a) original "Picnic" image, (b) Floyd-Steinberg algorithm with separable RGB palette, (c) Floyd-Steinberg algorithm with optimized palette, and (d) optimized error diffusion with optimized palette. (Halftones obtained from color images.)

56(6), 1009-1014 (1968).

20. M. A. Losada, R. Navarro, and J. Santamaria, "Relative contribution of optical and neural limitations to human contrast sensitivity at different luminance levels,' Vision Research 33(16), 2321-2336 (1993).

21. T. Mitsa and K. Varkur, "Evaluation of contrast sensitivity functions for the formulation of quality measures incorporated in halftoning algorithms,' Proc. IEEE Int'l. Conf. Acoustic Speech Signal Process., 5, 301-304, Minneapolis, MN (Apr. 27-30, 1993).
22. J. Sullivan, L. Ray, and R. Miller, "Design of minimum visual modulation halftone patterns," IEEE Trans. Syst., Man., Cybernetics, 21(1), 33-38 (1991).

23. J. Sullivan, R. Miller, and G. Pios, "Image halftoning using visual error diffusion," J. Opt. Soc. Am. A. 10(8), 1714-1724 (1993).

24. K. T. Mullen, "The contrast sensitivity of human color vision to redgreen and blue-yellow chromatic gratings," J. Physiol. 359, 381-400 (1985). 


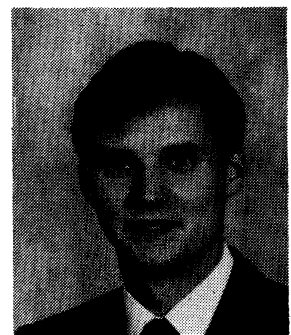

Bernd W. Kolpatzik received a Dipl-Ing in electrical engineering from the RuhrUniversität Bochum, Germany, in 1989 and the PhD from the School of Electrical Engineering at Purdue University, West Lafayette, Indiana, in 1993. In, October 1993, he joined the Image Science Lab at Polaroid Corporation in Cambridge, Massachusetts. His research interests include color image processing and display, visual models, and halftoning. He is a member of IEEE, IS\&T, and Tau Beta Pi.

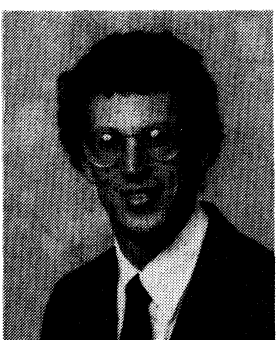

Charles A. Bouman received a BSEE degree from the University of Pennsylvania in 1981, and a MS degree in electrical engineering from the University of California at Berkeley in 1982. From 1982 to 1985 , he was a staff member in the Analog Device Technology Group at the Massachusetts Institute of Technology, Lincoln Laboratory. In 1987 and 1989 , he received MA and $\mathrm{PhD}$ degrees in electrical engineering from Princeton University under the support of an IBM graduate fellowship. In 1989, he joined the faculty of the School of Electrical Engineering at Purdue University as an assistant professor. Professor Bouman's research interests include statistical image modeling, multiscale processing, and the display and printing of images. He is particularly interested in the applications of statistical signal processing techniques to problems such as color halftoning, tomographic reconstruction, multispectral segmentation, and fast image search. Professor Bouman has performed research for numerous government and industrial organizations including the National Science Foundation, US Army, HewlettPackard, NEC Corporation, Apple Computers, Xerox, and Eastman Kodak. From 1991 to 1993 , he was also an NEC Faculty Fellow. Professor Bouman is a member of IEEE, SPIE, and IS\&T. He has been both chapter chair and vice chair of the IEEE Central Indiana Signal Processing Chapter. Currently, he is an associate editor of the IEEE Transactions on Image Processing and a member of the 1996 Image and Multidimensional Signal Processing organizing committee. 